Article

\title{
Evaluation of Automated Ribosomal Intergenic Spacer Analysis for Bacterial Fingerprinting of Rumen Microbiome Compared to Pyrosequencing Technology
}

\author{
Elie Jami ${ }^{1,2}$, Naama Shterzer ${ }^{1}$ and Itzhak Mizrahi ${ }^{1, *}$ \\ 1 Department of Ruminant Science, Institute of Animal Sciences, Agricultural Research Organization, \\ Volcani Center, PO Box 6, Bet Dagan 50250, Israel; E-Mails: eliejamie@gmail.com (E.J.); \\ naamash@volcani.agri.gov.il (N.S.) \\ 2 Department of Molecular Microbiology and Biotechnology, The George S. Wise Faculty of Life \\ Science, Tel Aviv University, Ramat-Aviv 69978, Israel \\ * Author to whom correspondence should be addressed; E-Mail: itzhakm@volcani.agri.gov.il; \\ Tel.: +972-3-9683026; Fax: +972-3-9683027.
}

Received: 24 November 2013; in revised form: 18 December 2013 / Accepted: 9 January 2014 / Published: 22 January 2014

\begin{abstract}
The mammalian gut houses a complex microbial community which is believed to play a significant role in host physiology. In recent years, several microbial community analysis methods have been implemented to study the whole gut microbial environment, in contrast to classical microbiological methods focusing on bacteria which can be cultivated. One of these is automated ribosomal intergenic spacer analysis (ARISA), an inexpensive and popular way of analyzing bacterial diversity and community fingerprinting in ecological samples. ARISA uses the natural variability in length of the DNA fragment found between the $16 \mathrm{~S}$ and $23 \mathrm{~S}$ genes in different bacterial lineages to infer diversity. This method is now being supplanted by affordable next-generation sequencing technologies that can also simultaneously annotate operational taxonomic units for taxonomic identification. We compared ARISA and pyrosequencing of samples from the rumen microbiome of cows, previously sampled at different stages of development and varying in microbial complexity using several ecological parameters. We revealed close agreement between ARISA and pyrosequencing outputs, especially in their ability to discriminate samples from different ecological niches. In contrast, the ARISA method seemed to underestimate sample richness. The good performance of the relatively inexpensive ARISA
\end{abstract}


makes it relevant for straightforward use in bacterial fingerprinting analysis as well as for quick cross-validation of pyrosequencing data.

Keywords: ARISA; pyrosequencing; rumen microbiome

\section{Introduction}

Analysis of bacterial diversity in environmental samples has always been a challenge for microbiologists. This field has been around for decades - ever since microscopy and culture-isolation methods were first implemented - but accurate assessment of the composition of an environmental sample has been, until recently, limited by our inability to culture most of the bacteria found in a given environment [1]. In the last decade however, with the improvement of high-throughput technologies and bioinformatic tools for analysis, many methods for the characterization of microbial diversity in complex environments have emerged. One such popular method, introduced by Fisher and Triplett (1999) [2], is automated ribosomal intergenic spacer analysis (ARISA). This method amplifies the DNA fragment found between the $16 \mathrm{~S}$ and $23 \mathrm{~S}$ genes in bacterial genomes and uses its natural variability in length - and separation by a capillary electrophoresis system (such as Sanger sequencing technology) - to infer diversity, with different sizes representing different operational taxonomic units (OTUs). This method is thought to describe the bacterial community at species level resolution [3], but has been shown to have some limitations in terms of accurately depicting microbial diversity in samples. A study by Kovacs et al. (2010) [3] revealed that with increasing species diversity, the method tends to underestimate species richness. This is the result of the limited fragment lengths that could be detected by this method, which range from 200 to $1,150 \mathrm{bp}$, restricting the number of different observable phylotypes within a sample to several hundreds. This means that ARISA might not be the most adequate assessment method for comparisons between samples with high taxon richness [4-7]. Nevertheless, ARISA has been extensively used for bacterial community analyses along with other non-sequencing methods such as DGGE or TRFLP [8-13], and a comparative study have found that it performed better than the other mentioned methods [9]. Another important aspect of community analysis lies in the ability of the fingerprinting method to accurately discriminate between samples for comparative analyses between different environments or conditions. In this context, ARISA was used to detect changes in bacterial community composition of a highly complex microbial environment residing in the rumen - the upper digestive tract compartment found in all ruminants [14-17]. Despite all of its benefits, ARISA falls short in comparison to the robust and now more accessible sequencing technologies. The latter's only limitation is the depth in which the samples are sequenced, thus it is less prone to underestimate sample richness. Additionally, pyrosequencing provides taxonomic identification by making use of the very comprehensive 16S rRNA databases available online [18]. Although the pyrosequencing methods are dropping in price, they remain more expensive than the fingerprinting methods. Furthermore, microbial ecology studies usually do not have an intrinsic reference point for control of the method itself [19]. Therefore, due to the different approach used with ARISA for microbial community analyses, this method, and its other fingerprinting counterpart could provide a quick and cost effective cross-validation procedure for pyrosequencing 
results, as previously reported [10,20]. Given its reported limitation with highly complex samples, in this study we aimed to systematically compare ARISA to pyrosequencing in rumen samples from cows at different ages, containing different levels of complexity and exhibiting high and low $\alpha$-diversity estimates, as previously observed by pyrosequencing [21]. We assessed the degree of agreement between the two methods, in terms of their ability to describe bacterial diversity and correctly discriminate between the sampling groups.

\section{Comparison of Local Richness Obtained from ARISA vs. Pyrosequencing}

We collected the rumen contents of 21 animals from different age groups: 1-3-day-old calves (three 1 -day-olds and three 3-day-olds, $\mathrm{n}=6)$, 2-month-old calves $(\mathrm{n}=5), 6$-month-old heifers $(\mathrm{n}=5)$ and 24-month-old lactating dairy cows $(n=5)$. These groups were fed according to conventional husbandry feeding programs for each age at our facility. Microbial cells were separated and metagenomic DNA was extracted as previously described [22,23]. We then used both bacterial tag-encoded amplicon pyrosequencing generated from the V2 and V3 regions of the 16S rRNA gene and ARISA to characterize the overall bacterial diversity in each of our samples. These samples were used in a previous study for the characterization of rumen bacterial composition across different ages [21]. In that study, the $\alpha$-diversity in the samples was found to increase with the age of the animal [21], thereby enabling a comparison of the ecological estimates of ARISA with those of pyrosequencing in samples with different complexities. As we previously reported, after size-filtering, quality control and chimera removal using the QIIME pipeline [24], a total of 227,414 quality reads were generated from the pyrosequencing effort with an average of 10,800 $\pm 2,860$ reads per sample. The overall number of OTUs detected by the pyrosequencing analysis was 6,594 based on $\geq 97 \%$ nucleotide-sequence identity between reads. The total number of OTUs associated with each age group, and their respective average OTU numbers per sample are reported in Table 1. Note that the samples from 2-year-old and 6-month-old animals exhibited different total possible OTU numbers and significant differences in OTU number per sample $(P<0.05$ using $t$-test analysis). Whole-community assessment using ARISA was performed using GeneMarker (SoftGenetics, USA) for ARISA resolution and noise filtering as previously carried out in previous studies [2,16]. The analysis revealed an overall lower bacterial diversity and a significantly lower average OTU number per sample as compared to the pyrosequencing (Table 1), with a total of 341 OTUs detected by ARISA. ARISA showed statistically significant discrimination between each age group-except between the 6-month-old and 2-year-old samples - when OTU numbers where compared, whereas the pyrosequencing method discriminated between all groups based on the same parameter (Table $1, P<0.05$ using $t$-test). However, Shannon diversity was significantly different between each group using the pyrosequencing data, whereas the ARISA data only discriminated between the 1-3-day-old samples and the rest of the samples, but not between the older age groups.

\section{3. $\beta$-Diversity Calculation}

The pairwise similarity within each group was calculated using the Bray-Curtis index, calculated from both the pyrosequencing and ARISA datasets, and the statistical significance of the changes in average similarity between groups was calculated as well (Figure 1). The ARISA average similarity 
values were consistently higher than those based on pyrosequencing for all groups $(P<0.05)$, However there was a strong correlation between ARISA and pyrosequencing values for each sample similarity (Figure 2; Pearson $\mathrm{R}=0.8, P<0.001$ ). We used analysis of similarity (ANOSIM) to assess whether the groups are indeed separate from one another using the pairwise similarity values. For both pyrosequencing and ARISA, all of the groups were significantly different from each other (Tables 2, 3). Each distance matrix of the samples for both ARISA and pyrosequencing were plotted using principal coordinate analysis (PCoA) (Figure 3a, b). The two methods clearly showed age group-based clustering with the two older groups clustering closer together, while the 2-month-olds and 1-3-day-olds clustered only within their own group. ANOSIM for both methods showed that the groups were indeed distinct from one another for both the pyrosequencing and ARISA data (Tables 2 and 3). Interestingly, the ARISA data separated the samples taken from 6-month-olds and those taken from 2-year-olds more clearly. Using Procrustes transformation, available in the QIIME package [24], two different PCoAs of the same samples can be superimposed by using different sets of data for analysis, and the degree of agreement between the two different sets of information for the same samples can be compared [25]. The transformation was performed on the three-dimensional principal coordinate analysis (PCoA) resulting from the pyrosequencing and ARISA data using Bray-Curtis as the distance index (Figure 3c). The goodness of fit $\left(\mathrm{M}^{2}\right)$ was calculated for the first three dimensions and was 0.45 with $P<0.00001$ based on 1,000 Monte-Carlo permutations. This revealed that the methods reached strong agreement in the clustering of the samples, allowing similar conclusions to be drawn about the degree of bacterial similarity and diversity between the samples and their respective groups (Figure 3).

Figure 1. Average Bray-Curtis similarity comparison between ARISA and pyrosequencing for each age group. Light-gray bars: pyrosequencing-based similarity values; dark-gray bars: ARISA-based similarity values.

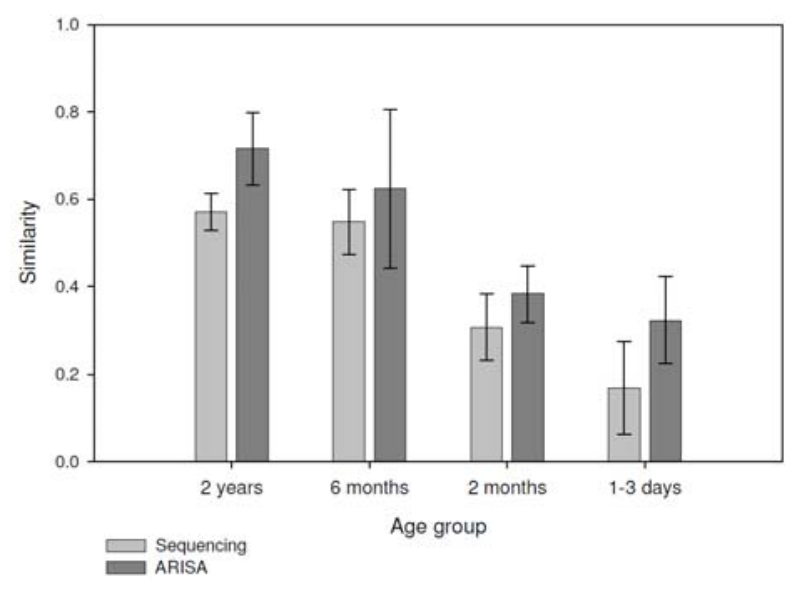

\section{Discussion}

Up until the recent rise in the use of next-generation pyrosequencing for bacterial fingerprinting, ARISA was a popular method of studying community diversity in gut samples, and was considered more accurate than other non-sequencing PCR-based community-analysis methods, such as TRFLP or LH-PCR $[9,11]$. We evaluated the sensitivity and accuracy of ARISA compared to community analysis based on $16 \mathrm{~S}$ whole-community pyrosequencing. The fact that these samples originated from the gut 
environment using identical sampling and extraction procedures allowed us to compare the performance of these two methods on various complexity measures using different ecological parameters. The number of OTUs revealed by the ARISA was significantly lower than that obtained with the pyrosequencing analysis based on 97\% similarity. In addition, using ARISA there were no statistical differences in OTU numbers or diversity metrics between the more complex samples (6-month-olds and 2-year-olds), making it less sensitive in discriminating richness between different environments or conditions. Pyrosequencing, on the other hand, did reveal a difference between the 2-year-old and 6-month-old samples. This could be explained by the lower sensitivity of the ARISA method, which underestimates richness in extremely rich environments as a result of a limited dynamic range and detection threshold, resulting from noise ratio for fluorescent fingerprinting techniques requiring to discard all signals under $0.1 \%$ abundance $[3,26]$. Additionally, it was previously observed that different bacterial taxa may have similar intergenic spacer length, or that the same taxa might have more than one ITS sequence length, further skewing sample richness [3,27,28]. Consequently, ARISA may not be an appropriate tool for assessing species richness within complex samples compared to the pyrosequencing method. Recent studies showed comparable underestimations of the bacterial community richness when ARISA was compared to pyrosequencing, ranging from 10 to 100 times lower OTU numbers [4,7,29]. However, both the ARISA and pyrosequencing data generated $\beta$-diversity estimates significantly discriminated between the different groups tested in a similar manner [7]. This held true in the current study as well in terms of relative diversity and similarity, as reflected by Procrustes analysis, ANOSIM and within-group similarity values (Figures 1, 2, and Tables 2,3$)$. In contrast to $\alpha$-diversity, $\beta$-diversity using the Bray-Curtis index managed to differentiate between the high-complexity samples in both methods, using the compositional and abundance differences of the observed OTUs. within this respect, ARISA demonstrated sharper discrimination between the 6-month-old and 2-years-old animals, compared to the higher degree of similarity between the groups observed using pyrosequencing. This highlights the comparable ability of ARISA to discriminate between different environments based on OTU composition despite its lower resolution, as also observed recently in samples of coastal sand bacterial communities [7].

Figure 2. Regression plot for each similarity measurement. Each point represents the similarity comparison between two samples from sequencing (Y-axis) and ARISA (X-axis). The $\mathrm{R}^{2}$ for the plot is 0.6 .

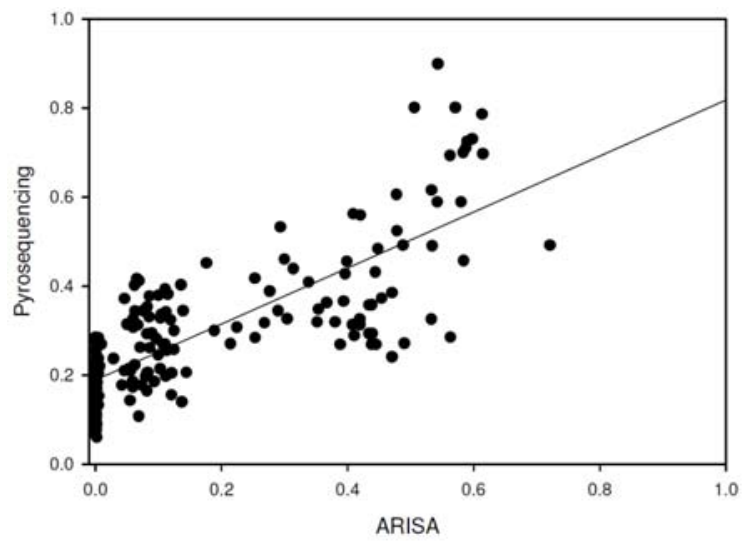


Figure 3. Distance ordination. Principal coordinate analysis (PCoA) plots were generated using the pairwise distance values for each sample using the Bray-Curtis metric. Every point in the plots represents the community in a single sample from either $16 \mathrm{~S}$ pyrosequencing or ARISA data and is colored according to the animal's age group (red, 1 day old; green, 3 days old; blue, 2 months old; orange, 6 months old, and purple, 2 years old). (a) PCoA of the distances resulting from the sequencing data. (b) PCoA of the distances resulting from the ARISA data. (c) Procrustes transformation analysis of 16S rRNA sequences against the ARISA-based operational taxonomic unit (OTU) clustering. The orange end of each line connects to the 16S rRNA data for the sample, and the black end of the line is connected to ARISA data for the sample.

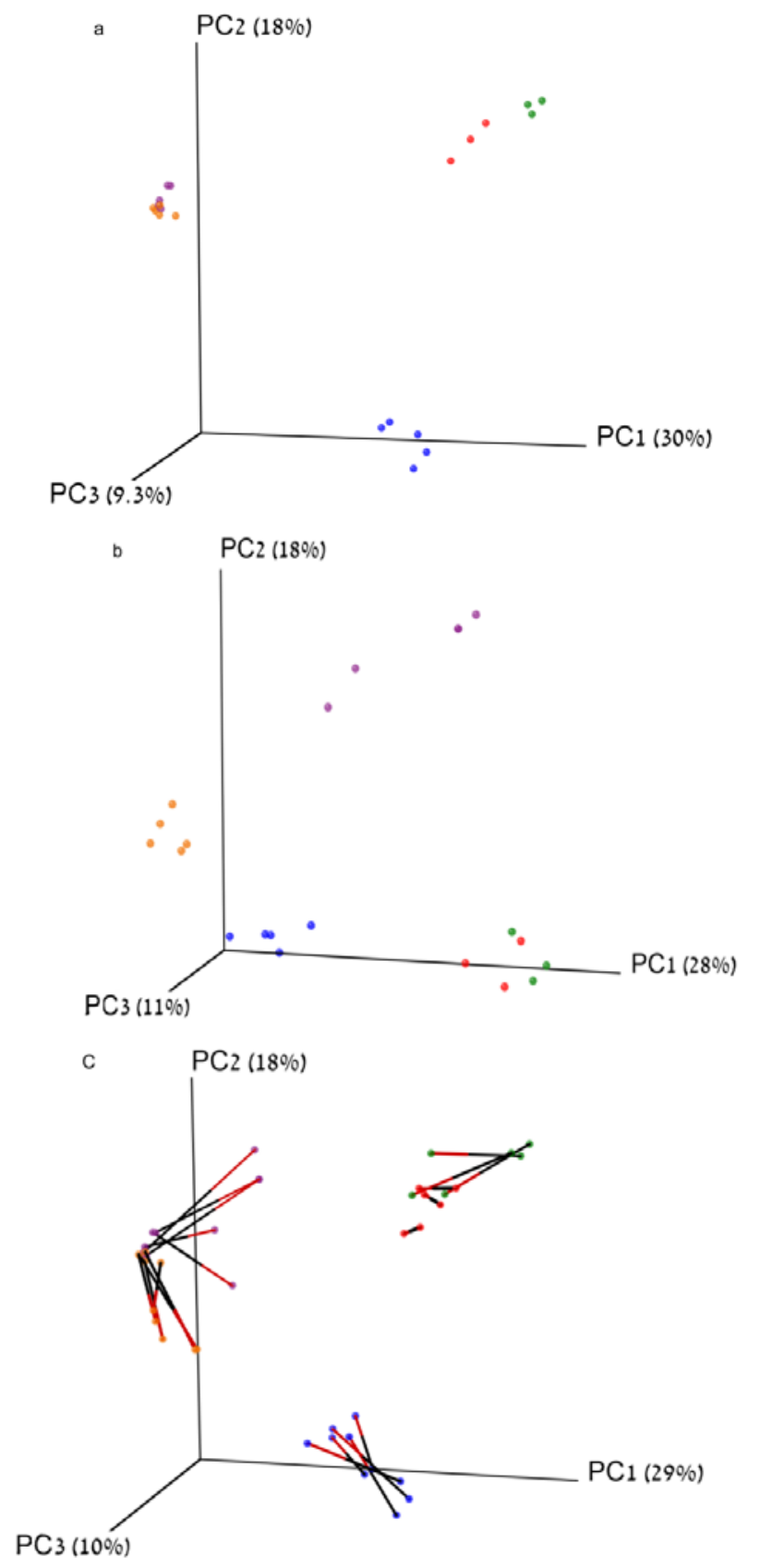


Table 1. Number of OTUs observed (OTU $\geq 97 \%$ considered species level) and Shannon-Wiener diversity for each age group, using ARISA and pyrosequencing results. Values in the same column with different superscript letters are significantly different $(P<0.01)$.

\begin{tabular}{|c|c|c|c|c|}
\hline & \multicolumn{2}{|c|}{$\begin{array}{c}\text { Number of OTUs identified (number of } \\
\text { OTUs per sample) }\end{array}$} & \multicolumn{2}{|c|}{$\begin{array}{c}\text { Shannon-Wiener }\left(H^{\prime}\right) \text { diversity } \\
\text { (SD) }\end{array}$} \\
\hline & Pyrosequencing & ARISA & Pyrosequencing & ARISA \\
\hline 1-3 days old & $380\left(208 \pm 47^{a}\right)$ & $206\left(90 \pm 18^{a}\right)$ & $2.8(0.49)^{\mathrm{a}}$ & $3.7(0.31)^{\mathrm{a}}$ \\
\hline 2 months old & $1441\left(620 \pm 100^{b}\right)$ & $204\left(116 \pm 8^{b}\right)$ & $3.7(0.36)^{b}$ & $4.2(0.15)^{b}$ \\
\hline 6 months old & $4074\left(2051 \pm 210^{c}\right)$ & $235\left(141 \pm 20^{\mathrm{c}}\right)$ & $6.2(0.3)^{\mathrm{c}}$ & $4.2(0.23)^{b}$ \\
\hline 2 years old & $4885\left(2382 \pm 263^{d}\right)$ & $214\left(148 \pm 23^{c}\right)$ & $6.5(0.08)^{\mathrm{d}}$ & $4.4(0.35)^{\mathrm{b}}$ \\
\hline
\end{tabular}

Table 2. Analysis of similarity between the age groups, based on the pairwise distance between samples, obtained using the pyrosequencing data. The R-values between each group are displayed in the lower left part of the table. The values in bold in the upper right part of the table represent the Bonferroni corrected $P$-values obtained between the groups.

\begin{tabular}{ccccc}
\hline Pyrosequencing & 2 years & 6 months & 2 months & 1-3 days \\
\hline 2 years & 0 & 0.043 & 0.042 & 0.01 \\
6 months & 0.916 & 0 & 0.034 & 0.012 \\
2 months & 1 & 1 & 0 & 0.015 \\
1-3 days & 1 & 1 & 1 & 0 \\
\hline
\end{tabular}

Table 3. Analysis of similarity between the age groups based on the pairwise distance between samples obtained using the ARISA data. The R-values between each group are displayed in the lower left part of the table. The values in bold in the upper right part of the table represent the Bonferroni corrected $P$-values obtained between the groups.

\begin{tabular}{ccccc}
\hline ARISA & 2 years & 6 months & 2 months & 1-3 days \\
\hline 2 years & 0 & 0.047 & 0.047 & 0.012 \\
6 months & 0.96 & 0 & 0.047 & 0.013 \\
2 months & 0.684 & 0.948 & 0 & 0.012 \\
1-3 days & 0.9387 & 0.7893 & 0.984 & 0 \\
\hline
\end{tabular}

\section{Experimental Section}

\subsection{Animal Handling and Sampling}

The experimental procedures used in this study were approved by the Faculty Animal Policy and Welfare Committee of the Agricultural Research Organization (ARO), approval number IL-168/08, Volcani Research Center, and were in accordance with the guidelines of the Israel Council on Animal Care.

Israeli Holstein Friesian lactating cows and heifers $(n=21)$ were housed at the ARO's experimental dairy farm in Bet Dagan, Israel. Pre-weaned calves were housed separately, while adult animals were housed in one shaded corral with free access to water. The cows were fed according to the conventional feeding regimen at our farm [21]. The samples were taken $1 \mathrm{~h}$ after the morning feeding: ruminal contents were collected via the cow's mouth $-500 \mathrm{~mL}$ from adults and $100 \mathrm{~mL}$ from young 
calves - using a stainless-steel stomach tube with a rumen vacuum sampler. Samples were transferred to $\mathrm{CO}_{2}$-containing centrifuge bottles to maintain anaerobic conditions, and kept on ice. After collection, the samples were processed in the laboratory adjacent to the farm.

\subsection{Isolation of Microbial Fraction from the Rumen}

The microbial fraction was isolated according to [22] with the modifications described by Jami and Mizrahi (2012) [17]. Briefly, following $2 \mathrm{~min}$ of blender homogenization, we centrifuged the rumen samples at $10,000 \mathrm{~g}$ for $30 \mathrm{~min}$ at $4{ }^{\circ} \mathrm{C}$ and dissolved the pellet in extraction buffer $(100 \mathrm{mM}$ Tris- $\mathrm{HCl}, 10 \mathrm{mM}$ ethylenediaminetetraacetic acid [EDTA], $0.15 \mathrm{M} \mathrm{NaCl} \mathrm{pH}$ 8.0). The suspension was held at $4{ }^{\circ} \mathrm{C}$ for $1 \mathrm{~h}$ to maximize the release of particle-associated bacteria from the ruminal contents [22]. The suspension was then gently centrifuged at $500 \mathrm{~g}$ for $15 \mathrm{~min}$ at $4{ }^{\circ} \mathrm{C}$ to remove ruptured plant particles while keeping the bacterial cells in suspension [30]. The supernatant was filtered through four layers of cheesecloth and centrifuged $\left(10,000 \mathrm{~g}, 25 \mathrm{~min}, 4{ }^{\circ} \mathrm{C}\right)$, and the pellets were kept at $-20^{\circ} \mathrm{C}$ until DNA extraction.

\subsection{DNA Extraction}

DNA extraction was performed by bead disruption with phenol, followed by phenol/chloroform DNA extraction as described by [22]. Isopropanol was then used for precipitation $(0.6: 1 \mathrm{v} / \mathrm{v})$ and the precipitate was resuspended in 50 to $100 \mu \mathrm{L}$ Tris-EDTA buffer, then stored at $4{ }^{\circ} \mathrm{C}$ for short-term use, or archived at $-20^{\circ} \mathrm{C}$.

\subsection{Automated Ribosomal Intergenic Spacer Analysis (ARISA)}

DNA from all rumen samples was subjected to PCR amplification for ARISA [2]. The oligonucleotide primers ITSF (5'-GTCGTAACAAGGTAGCCGTA-3') and ITSRtet (5'-GCCAAGGCATCCAAC-3') were used for ARISA of rumen bacteria, with the fluorescent molecule TET used as described recently by [15]. ARISA PCRs were carried out in $15-\mu \mathrm{L}$ volumes containing Fermentas Dreamtaq (Madison, WI) master mix, $0.5 \mu \mathrm{L}$ of $10 \mu \mathrm{M}$ stock solution for each primer, $20 \mathrm{ng}$ of template DNA and $4.5 \mu \mathrm{L}$ of nuclease-free water. PCR was carried out using a Sensiquest thermocycler (Gottingen, Germany) under the following conditions: $94{ }^{\circ} \mathrm{C}$ for $2 \mathrm{~min}$ ( 1 cycle), followed by 30 cycles of $94{ }^{\circ} \mathrm{C}$ for $1 \mathrm{~min}, 55^{\circ} \mathrm{C}$ for $60 \mathrm{~s}$ and $72{ }^{\circ} \mathrm{C}$ for $120 \mathrm{~s}$, and finally 1 cycle at $72{ }^{\circ} \mathrm{C}$ for $5 \mathrm{~min}$.

\subsection{ARISA Resolution and Analysis}

For each DNA sample, two technical replicates of PCR products were analyzed using an ABI PRISM 3,100 Genetic Analyzer. The labeled fragments were separated on the capillary sequencer along with a custom-made ROX-labeled 250- to 1,150-bp standard (Bioventures). Raw data generated by the genetic analyzer were initially analyzed using GeneMarker (Softgenetics, USA) according to [3]. After performing accurate size calling using the program, all data were exported to Microsoft Excel for further analysis. All OTUs with fluorescence intensity of $\leq 10 \mathrm{RFU}$ (relative fluorescence units) were excluded. The remaining OTUs were binned as described by [27] with the following parameters: bins of $3 \mathrm{bp}$ ( $\pm 1 \mathrm{bp}$ ) for fragments up to $700 \mathrm{bp}$ in length, bins of $5 \mathrm{bp}$ for fragments between 700 and 1,000 bp 
in length, and bins of $10 \mathrm{bp}$ for fragments longer than 1,000 bp. Intensities were then summed for each bin. Next, relative intensities for each binned OUT in a given sample were calculated and OTUs, which constituted less than $0.1 \%$ of the total intensity of the sample, were excluded. Technical duplicates were compared as individual samples and served as an internal control for the quality of the analysis. Duplicates that were not similar to each other were further checked for technical-run problems and were either discarded or run again.

\subsection{Tag Amplicon Pyrosequencing and Data Analyses}

454 Amplicon pyrosequencing of the ruminal DNA samples was performed by the Research and Testing Laboratory (Lubbock, TX) using primers covering the 103- to 530-bp region of the 16S rRNA gene sequence which corresponds to the V2 and V3 regions (107F: 5'-GGCGVACGGGTGAGTAA-3' and 530R: 5'-CCGCNGCNGCTGGCAC-3'). The tagging and sequencing protocol was as described by [31]. Data quality control and analyses were mostly performed using the QIIME pipeline [24] and as described by Jami et al. (2013). Briefly, reads were assigned to their designated rumen sample, then length-based filtering $(<200 \mathrm{bp}$ was excluded from the analysis), read-quality filtering and chimeric-sequence removal [32] were performed. Binning of OTUs according to the predefined threshold of $>97 \%$ similarity was performed using the Uclust clustering method [33]. Clusters comprising only one (singletons) or two (doubletons) reads were removed.

\subsection{Statistical Analyses}

Procrustes analysis was performed in QIIME, and the two individual three-dimensional principal coordinates (PCoA) (one for each analytical method) were generated based on a distance matrix calculated using the Bray-Curtis index [34], transformed and visualized in a plot. Statistical analyses of the OTU data were performed using PAleontological STatistics (PAST) software [35], including diversity indexes, correlations and ANOSIM.

\section{Conclusion}

ARISA proved to be a reliable tool for the discrimination of samples based on $\beta$-diversity data. Nevertheless, it exhibited some limitations in estimating $\alpha$-diversity in highly complex samples, as compared to the pyrosequencing method. Despite the decreasing cost of pyrosequencing and the increasing demand in taxonomy for community assessments, ARISA remains an accurate and relevant method for comparing different environments and/or different treatments, with the added advantage of still being considerably less expensive than pyrosequencing. Moreover, it can serve as a good cross-validation procedure for pyrosequencing techniques.

\section{Conflicts of Interest}

The authors declare no conflict of interest. 


\section{References}

1. Janssen, P.H. Identifying the dominant soil bacterial taxa in libraries of 16s rRNA and 16s rRNA genes. Appl. Environ. Microbiol. 2006, 72, 1719-1728.

2. Fisher, M.M.; Triplett, E.W. Automated approach for ribosomal intergenic spacer analysis of microbial diversity and its application to freshwater bacterial communities. Appl. Environ. Microbiol. 1999, 65, 4630-4636.

3. Kovacs, A.; Yacoby, K.; Gophna, U. A systematic assessment of automated ribosomal intergenic spacer analysis (arisa) as a tool for estimating bacterial richness. Res. Microbiol. 2010, 161, 192-197.

4. Koopman, M.M.; Fuselier, D.M.; Hird, S.; Carstens, B.C. The carnivorous pale pitcher plant harbors diverse, distinct, and time-dependent bacterial communities. Appl. Environ. Microbiol. 2010, 76, 1851-1860.

5. Bent, S.J.; Forney, L.J. The tragedy of the uncommon: Understanding limitations in the analysis of microbial diversity. ISME J. 2008, 2, 689-695.

6. Bent, S.J.; Pierson, J.D.; Forney, L.J.; Danovaro, R.; Luna, G.M.; Dell'sanno, A.; Pietrangeli, B. Measuring species richness based on microbial community fingerprints: The emperor has no clothes. Appl. Environ. Microbiol. 2007, 73, 2399-2401.

7. Gobet, A.1.; Boetius, A.; Ramette, A. Ecological coherence of diversity patterns derived from classical fingerprinting and next generation sequencing techniques. Environ. Microbiol. 2013, doi:10.1111/1462-2920.12308.

8. Brusetti, L.; Borin, S.; Rizzi, A.; Mora, D.; Sorlini, C.; Daffonchio, D. Exploration of methods used to describe bacterial communities in silage of maize (zea mays) cultivars. Environ. Biosafety. Res. 2008, 7, 25-33.

9. Danovaro, R.; Luna, G.M.; Dell'anno, A.; Pietrangeli, B. Comparison of two fingerprinting techniques, terminal restriction fragment length polymorphism and automated ribosomal intergenic spacer analysis, for determination of bacterial diversity in aquatic environments. Appl. Environ. Microbiol. 2006, 72, 5982-5989.

10. Gillevet, P.M.; Sikaroodi, M.; Torzilli, A.P. Analyzing salt-marsh fungal diversity: Comparing arisa fingerprinting with clone sequencing and pyrosequencing. Fungal Ecology 2009, 2, 160-167.

11. Mills, D.K.; Fitzgerald, K.; Litchfield, C.D.; Gillevet, P.M. A comparison of DNA profiling techniques for monitoring nutrient impact on microbial community composition during bioremediation of petroleum-contaminated soils. J. Microbiol. Methods 2003, 54, 57-74.

12. Sikaroodi, M.; Gillevet, P.M. Quality control in multi-tag pyrosequencing of microbial communities. Biotechniques 2012, 53, 381-383.

13. Shade, A.; Read, J.S.; Youngblut, N.D.; Fierer, N.; Knight, R.; Kratz, T.K.; Lottig, N.R.; Roden, E.E.; Stanley, E.H.; Stombaugh, J.; et al. Lake microbial communities are resilient after a whole-ecosystem disturbance. ISME J. 2012, 6, 2153-2167.

14. Jami, E.; Shabtay, A.; Nikbachat, M.; Yosef, E.; Miron, J.; Mizrahi, I. Effects of adding a concentrated pomegranate-residue extract to the ration of lactating cows on in vivo digestibility and profile of rumen bacterial population. J. Dairy Sci. 2012, 95, 5996-6005.

15. Welkie, D.G.; Stevenson, D.M.; Weimer, P.J. Arisa analysis of ruminal bacterial community dynamics in lactating dairy cows during the feeding cycle. Anaerobe 2009, 16, 94-100. 
16. Weimer, P.J.; Stevenson, D.M.; Mertens, D.R.; Thomas, E.E. Effect of monensin feeding and withdrawal on populations of individual bacterial species in the rumen of lactating dairy cows fed high-starch rations. Appl. Microbiol. Biotechnol. 2008, 80, 135-145.

17. Jami, E.; Mizrahi, I. Similarity of the ruminal bacteria across individual lactating cows. Anaerobe 2012, 18, 338-343.

18. DeSantis, T.Z.; Hugenholtz, P.; Larsen, N.; Rojas, M.; Brodie, E.L.; Keller, K.; Huber, T.; Dalevi, D.; $\mathrm{Hu}$, P.; Andersen, G.L. Greengenes, a chimera-checked 16s rRNA gene database and workbench compatible with ARB. Appl. Environ. Microbiol. 2006, 72, 5069-5072.

19. Bokulich, N.A.; Subramanian, S.; Faith, J.J.; Gevers, D.; Gordon, J.I.; Knight, R.; Mills, D.A.; Caporaso, J.G. Quality-filtering vastly improves diversity estimates from illumina amplicon sequencing. Nat. Methods 2013, 10, 57-59.

20. Gillevet, P.; Sikaroodi, M.; Keshavarzian, A.; Mutlu, E.A. Quantitative assessment of the human gut microbiome using multitag pyrosequencing. Chem. Biodivers. 2010, 7, 1065-1075.

21. Jami, E.; Israel, A.; Kotser, A.; Mizrahi, I. Exploring the bovine rumen bacterial community from birth to adulthood. ISME J. 2013, 7, 1069-1079.

22. Stevenson, D.M.; Weimer, P.J. Dominance of prevotella and low abundance of classical ruminal bacterial species in the bovine rumen revealed by relative quantification real-time pcr. Appl. Microbiol. Biotechnol. 2007, 75, 165-174.

23. Jami, E.; Mizrahi, I. Composition and similarity of bovine rumen microbiota across individual animals. PLoS One 2012, 7, e33306.

24. Caporaso, J.G.; Kuczynski, J.; Stombaugh, J.; Bittinger, K.; Bushman, F.D.; Costello, E.K.; Fierer, N.; Pena, A.G.; Goodrich, J.K.; Gordon, J.I.; et al. Qiime allows analysis of high-throughput community sequencing data. Nat Methods 2011, 7, 335-336.

25. Muegge, B.D.; Kuczynski, J.; Knights, D.; Clemente, J.C.; Gonzalez, A.; Fontana, L.; Henrissat, B.; Knight, R.; Gordon, J.I. Diet drives convergence in gut microbiome functions across mammalian phylogeny and within humans. Science 2011, 332, 970-974.

26. Dunbar, J.; Ticknor, L.O.; Kuske, C.R. Phylogenetic specificity and reproducibility and new method for analysis of terminal restriction fragment profiles of $16 \mathrm{~s}$ rrna genes from bacterial communities. Appl. Environ. Microbiol. 2001, 67, 190-197.

27. Brown, M.V.; Schwalbach, M.S.; Hewson, I.; Fuhrman, J.A. Coupling 16s-its rdna clone libraries and automated ribosomal intergenic spacer analysis to show marine microbial diversity: Development and application to a time series. Environ. Microbiol. 2005, 7, 1466-1479.

28. Crosby, L.D.; Criddle, C.S. Understanding bias in microbial community analysis techniques due to rrn operon copy number heterogeneity. Biotechniques 2003, 34, 790-803.

29. Roesch, L.F.; Lorca, G.L.; Casella, G.; Giongo, A.; Naranjo, A.; Pionzio, A.M.; Li, N.; Mai, V.; Wasserfall, C.H.; Schatz, D.; et al. Culture-independent identification of gut bacteria correlated with the onset of diabetes in a rat model. ISME J. 2009, 3, 536-548.

30. Dehority, B.A.; Grubb, J.A. Effect of short-term chilling of rumen contents on viable bacterial numbers. Appl. Environ. Microbiol. 1980, 39, 376-381.

31. Dowd, S.E.; Callaway, T.R.; Wolcott, R.D.; Sun, Y.; McKeehan, T.; Hagevoort, R.G.; Edrington, T.S. Evaluation of the bacterial diversity in the feces of cattle using 16s rRNA bacterial tag-encoded flx amplicon pyrosequencing (btefap). BMC Microbiol. 2008, doi:10.1186/1471-2180-8-125. 
32. Haas, B.J.; Gevers, D.; Earl, A.M.; Feldgarden, M.; Ward, D.V.; Giannoukos, G.; Ciulla, D.; Tabbaa, D.; Highlander, S.K.; Sodergren, E.; et al. Chimeric 16s rRNA sequence formation and detection in sanger and 454-pyrosequenced pcr amplicons. Genome Res. 2011, 21, 494-504.

33. Edgar, R.C. Search and clustering orders of magnitude faster than blast. Bioinformatics 2010, 26, 2460-2461.

34. Bray, J.R.; Curtis, J.T. An ordination of the upland forest communities of southern wisconsin. Ecol. Monogr. 1957, 27, 325-349.

35. Hammer, Ø.; Harper, D.A.T.; Ryan, P.D. Past: Paleontological statistics software package for education and data analysis. Palaeontol. Electron. 2001, 4, 1-9.

(C) 2014 by the authors; licensee MDPI, Basel, Switzerland. This article is an open access article distributed under the terms and conditions of the Creative Commons Attribution license (http://creativecommons.org/licenses/by/3.0/). 